BULLETIN OF THE

AMERICAN MATHEMATICAL SOCIETY

Volume 80, Number 1, January 1974

\title{
GLOBAL DEFORMATION OF POLARIZED VARIETIES ${ }^{1}$
}

\author{
BY T. MATSUSAKA
}

1. Classically, the works of Riemann, Hurwitz, and Severi show that the set of complete nonsingular curves of genus $g$ can be parametrized into an algebraic family of nonsingular curves, parametrized by an irreducible algebraic variety (allowing many curves of the family to be isomorphic to each other), and that isomorphism classes of these curves depend on $3 g-3$ parameters $(g>1)$. Moreover, two complete nonsingular curves of different genera cannot be members of an algebraic family parametrized by an irreducible algebraic variety. These imply, among other things, that the topological as well as the differential geometric nature of such curves can be characterized completely by the genus. Moreover, it is known from the result of Baily [2], [3] that distinct isomorphism classes of such curves of genus $g$ form an irreducible algebraic variety of dimension $3 g-3$ ( 1 if $g=1$ ).

When we attempt to deal with similar problems for complete nonsingular and projectively embeddable varieties of higher dimensions, we encounter difficulties of higher magnitude. Limiting ourselves to the category of algebraic varieties for characteristic zero, Siegel [18], Satake [16] and Baily [3] have shown that similar results are true for polarized Abelian varieties. We call a pair $(V, \mathscr{X})$ of a complete nonsingular variety $V$ and a set $\mathscr{X}$ of $V$-divisors a polarized variety if $\mathscr{X}$ satisfies the following conditions:

(i) $\mathscr{X}$ contains a nondegenerate divisor $X$ (ample in the sense of [5]);

(ii) A $V$-divisor $Y$ is in $\mathscr{X}$ if and only if $r Y \equiv s X$ (numerical equivalence) for some integers $r, s$.

A divisor $Y$ in $\mathscr{X}$ is called a polar divisor. $\mathscr{X}$ contains a divisor $X_{0}$ such that a $V$-divisor $Y$ is in $\mathscr{X}$ if and only if $Y \equiv s X_{0}$ for some integer $s$ and a nondegenerate polar divisor $X$ can be expressed as $X \equiv r X_{0}$ with a positive integer $r$ (cf. [10]). Such a polar divisor $X_{0}$ is called a basic polar divisor. Already there are many examples of algebraic surfaces which indicate

This is a slightly expanded version of the invited talk given at a meeting of the Society in New York, April 20, 1973; received by the editors May 10, 1973.

AMS (MOS) subject classifications (1970). Primary 14-02, 14D15, 14D20, 14E25.

Key words and phrases. Polarized varieties, polar divisors, basic polar divisors, Hilbert characteristic polynomial of a polarized variety, moduli, $Q$-variety, rational map defined by a module of functions.

${ }^{1}$ The works discussed here have been done with partial support from NSF. 
that similar results as in the case of curves cannot be obtained without at least an additional structure of polarization. Then, is it possible, with the additional structure of polarization, to obtain similar results?

2. In order to describe our problem a little more in detail, let us recall the definition of the Hilbert characteristic polynomial of a polarized variety $(V, \mathscr{X})$ (cf. [11]). Let $X_{0}$ be a basic polar divisor of it. Its numerical equivalence class is uniquely determined and the polynomial $\chi\left(V, \mathscr{L}\left(m X_{0}\right)\right)$, the Euler-Poincare characteristic of $V$ with coefficients in the invertible sheaf determined by $m X_{0}$, is uniquely determined by our polarized variety. This polynomial is called the Hilbert characteristic polynomial of $(V, \mathscr{X})$. In the case of surfaces, the coefficients of this polynomial are completely determined by the self-intersection number $X_{0}^{(2)}$ of $X_{0}$, by the virtual arithmetic genus $p_{a}\left(X_{0}\right)$ of $X_{0}$ and by the arithmetic genus $p_{a}(V)$ of $V$. By relaxing our requirements a little, we can ask the following questions, corresponding to the case of curves and polarized Abelian varieties.

(I) Is it possible to parametrize the set of polarized varieties with a given Hilbert characteristic polynomial, up to isomorphisms, by a finite number of irreducible algebraic varieties?

(II) If the answer to (I) is affirmative, how many parameters do the isomorphism classes of such polarized varieties depend on?

(III) If the answer to (I) is affirmative, and when $U$ is an algebraic variety of finite type which parametrizes the family in (I), is it possible to construct the variety of moduli? (That is, when we introduce in $U$ an equivalence relation on points induced by isomorphisms of fibres, is it possible to find the quotient space of $U$ as an algebraic variety of finite type?)

Anticipating (III), we introduced the concept of $Q$-varieties (cf. [9]) which is a little wider in concept than algebraic varieties and on which some important algebro-geometric operations can be performed (e.g. intersection theory). Then we showed that once (I) is solved, (III) can be solved in the category of $Q$-varieties, if we exclude all ruled varieties (cf. M. Artin's work [1]). Whether a solution of (III) can be found in general within the category of algebraic varieties seems to be an unsolved question as far as we know. But the answer could quite possibly be negative. (II) is in general an unsolved problem, but in some important concrete cases solutions are given in [7]. Because of these situations, let us concentrate here on the solution of (I) and also on the case of characteristic zero.

3. The affirmative answer to (I) in the case of curves comes essentially from the Riemann-Roch theorem on curves and from Chow-v.d.Waerden's Chow form or from Grothendieck's Hilbert scheme (cf. [4], [5]). The answer to (I) for polarized Abelian varieties can be obtained when we 
replace the Riemann-Roch theorem above by a theorem of Lefschetz that $3 X$ is ample whenever $X$ is nondegenerate (cf. [8]). The case of a polarized surface was more difficult but we could settle it affirmatively, in our joint paper with Mumford, using many available results on nonsingular surfaces (cf. [14]). Especially, we found that the Riemann-Roch inequality on surfaces played an important, if not decisive, role.

In relation to (i), we can formulate and ask the following questions.

$\left(\mathrm{I}_{1}\right)$ Let $V$ be a complete nonsingular variety, $X$ a nondegenerate divisor on $V$ and $P(m)=\chi(V, \mathscr{L}(m X))$. Is it possible to find a constant $c$, depending only on the polynomial $P(x)$, such that $h^{i}(V, \mathscr{L}(m X))=0$ for $i>0$ if $m>c$ ?

$\left(\mathrm{I}_{2}\right)$ Let $V, X$ be as above. Is it possible to find a constant $c^{\prime}$, depending only on the polynomial $P(x)$, such that the module $L(m X)=H^{0}(V, \mathscr{L}(m X))$ defines a projective embedding if $m>c^{\prime}$ ?

We have solved $\left(I_{2}\right)$ which yields an affirmative answer to (I) by the aid of the main theorem on Chow-forms or Hilbert schemes. Also $\left(I_{1}\right)$ is a consequence of $\left(I_{2}\right)$. But a direct solution of $\left(I_{1}\right)$ is much desired. Not only would it simplify our proofs but it would also give deeper insight into the Riemann-Roch theorem even in the special case when $X$ is a nondegenerate divisor.

Lack of strong vanishing theorem $\left(\mathrm{I}_{1}\right)$ forced us to use a different but strong vanishing theorem of Kodaira: When $V$ is a nonsingular projective variety and $X$ a nondegenerate divisor on $V, h^{i}(V, \mathscr{L}(X+K(V)))=0$ for $i>0$, where $K(V)$ is a canonical divisor of $V$ (cf. [6]). This forced us to assume that the characteristic of the universal domain is zero. In relation to this, let us consider the following problem.

$\left(\mathrm{I}_{3}\right)$ Let $V$ be a complete nonsingular variety in characteristic $p$ and $X$ a nondegenerate $V$-divisor. Is it possible to find a constant $c^{*}$, depending only on the polynomial $\chi(V, \mathscr{L}(m X))$ such that $h^{i}(V, \mathscr{L}(X+K(V)))<c^{*}$ ? Or is it possible to find an absolute constant $c^{*}$ with the above property?

We are hopeful that we have found an idea to solve $\left(\mathrm{I}_{3}\right)$. As to the possibility of generalizing Kodaira's vanishing theorem as it is to the case of characteristic $p$, the chance looks rather dim (cf. [15]).

4. As to the detail of solutions of $\left(I_{2}\right)$ in the case of characteristic 0 , we refer the reader to my paper [13], which has appeared in the American Journal of Mathematics. We shall touch on the rough ideas very briefly.

When $U$ is a variety and $M$ a module of rational functions on $U$ of finite dimension, let $f_{0}, \cdots, f_{m}$ be a basis of $M$. Then $x \rightarrow\left(f_{0}(x): f_{1}(x): \cdots: f_{m}(x)\right)$ defines a rational map of $U$ into a projective space. We shall call this the rational map defined by $M$ which is unique up to a projective transformation. 
Let $V, X, P(x)$ be as in the problem $\left(\mathrm{I}_{2}\right)$. When the polynomial $P^{\prime}(x)$ is defined by

$$
P^{\prime}(m)=\chi(V, \mathscr{L}(m X+K(V))),
$$

$P(m)=(-1)^{n} P^{\prime}(-m)$ by the duality theorem of Serre (cf. [17]). From this we see that

$$
d=X^{(n)}, \quad \xi=I\left(X^{(n-1)}, K(V)\right)
$$

are uniquely determined by $P(x)$. Moreover, we can find a constant $c_{0}$, depending only on $P(x)$ such that for $m \geqq c_{0}, h^{0}(V, \mathscr{L}(m X+K(V)))=P^{\prime}(m)$, $P^{\prime}(m+1)>P^{\prime}(m)$. We first derive the inequalities

(1) $d u^{i}(m+\xi / d)^{i} \geqq h^{0}(V, \mathscr{L}(u m X+u K(V))) \geqq P^{\prime}\left(u\left(m-c_{0}\right)+c_{0}\right)$

for $m \geqq c_{0}$, provided that the rational map $f_{u, m}$ defined by the module $H^{0}(V, \mathscr{L}(u m X+u K(V)))$ maps $V$ on a variety of dimension $i$. From these, we see at once that if $m>\max \left(c_{0},|\xi| / d\right)$, there is a constant $c(m)$, depending only on $P(x)$ and $m$, such that $f_{u, m}$ maps $V$ on a variety of dimension $n$ if $u>c(m)$.

We then fix a positive integer $m_{0}>c_{0}$, such that

$$
2^{1^{1 / n}}\left(m-c_{0}\right) /(m+\xi / d)>1
$$

for $m>m_{0}$ and let the left hand side be $\geqq 1+\varepsilon_{0}$ with $\varepsilon_{0}>0$ for such $m$. Define $c_{1}$ by $c\left(m_{0}\right)$. Consider, for $s>c_{1}, m>0$ the following diagram

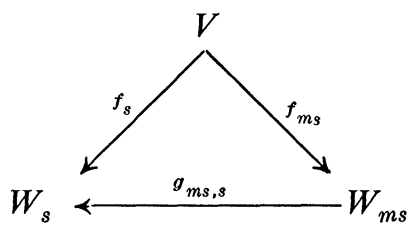

where $f_{s}=f_{s, m_{0}}, W_{s}$ the image of $V$ by $f_{s}$ and $g_{m s, s}$ the rational map which makes the above diagram generically commutative.

Assume, for a moment, that $\operatorname{deg}\left(f_{s}\right)>1, \operatorname{deg}\left(g_{m s, s}\right)=1$ and that

$$
\operatorname{deg}\left(g_{m s, s}(C)\right)<\left(1+\varepsilon_{0}\right) m \cdot \operatorname{deg}\left(C^{\prime}\right),
$$

where $C$ (resp. $C^{\prime}$ ) is a hyperplane section of $W_{m s}$ (resp. $W_{s}$ ). Then, we can find two polynomials $Q_{n}(y, x), Q_{n}^{\prime}(x)$ with nonnegative rational coefficients of degree $<n$ in $x$ and linear in $y$, determined uniquely by $P(x)$, such that

(2) $l\left(\operatorname{smm}_{0} X+\operatorname{smK}(V)\right)$

$$
<(\delta / n !)\left(1+\varepsilon_{0}\right)^{n} m^{n}+Q_{n}\left(\delta,\left(1+\varepsilon_{0}\right) m\right)+Q_{n}^{\prime}\left(\left(1+\varepsilon_{0}\right) m\right),
$$

where

$$
l\left(s m m_{0} X+\operatorname{smK}(V)\right)=h^{0}\left(V, \mathscr{L}\left(s m m_{0} X+\operatorname{sm} K(V)\right)\right)
$$


and $\delta=(d / 2) s^{n}\left(m_{0}+\xi / d\right)^{n}$. When we compare this with our first inequalities (1), we see the existence of a positive integer $e(s)$, which depends only on $P(x), \varepsilon_{0}$ and $s$ such that for $m \geqq e(s),(1)$ and (2) are incompatible and hence $\operatorname{deg}\left(g_{m s, s}(C)\right) \geqq\left(1+\varepsilon_{0}\right) m \cdot \operatorname{deg}\left(C^{\prime}\right)$ for such $m$. Using this result, we compare the degrees of $W_{s}$ and $W_{m s}$ under our assumptions and get

$$
\operatorname{deg} W_{m s} \geqq m^{n-1} \operatorname{deg}\left(g_{m s, s}(C)\right) \geqq m^{n}\left(1+\varepsilon_{0}\right) \cdot \operatorname{deg} W_{s},
$$

if $s>c_{1}$ and $m \geqq e(s)$. In general, we have the following inequality:

$$
d s^{n}\left(m_{0}+\xi / d\right)^{n} \geqq \operatorname{deg}\left(f_{s}\right) \cdot \operatorname{deg} W_{s}
$$

for $s>c_{1}$. This, together with the above inequalities show that $\operatorname{deg}\left(f_{s}\right)>1$, and the series of equalities

$$
\operatorname{deg}\left(g_{t_{1} \cdots t_{i} s, t_{1} \cdots t_{i-1} s}\right)=1 \text { with } t_{i}=e\left(s t_{1} \cdots t_{i-1}\right)
$$

cannot continue to hold indefinitely. When we select $r_{0}$ to be the least positive integer satisfying $d s^{n}\left(m_{0}+\xi / d\right)^{n}<\left(1+\varepsilon_{0}\right)^{r}$ for $r \geqq r_{0}$, the series of equalities stops when $i>r_{0}$. From this we can show that the following theorem is true.

THEOREM 1. There is a pair $\left(m_{0}, c_{2}\right), c_{2} \geqq c_{1}$, of positive integers, which can be determined by $P(x)$ alone, such that $H^{0}\left(V, \mathscr{L}\left(m m_{0} X+m K(V)\right)\right)$ defines a birational transformation of $V$ whenever $m \geqq c_{2}$.

5. Let, as before, $f_{m}$ be the rational map of $V$ into a projective space defined by the module $H^{0}\left(V, \mathscr{L}\left(m m_{0} X+m K(V)\right)\right)$ where $m \geqq c_{2}$. Then $f_{m}$ is a birational transformation. But there may be a subvariety $A$ of codimension 1 on $V$ such that its proper image $f_{m}[A]$ by $f_{m}$ has lesser dimension than $n-1$. Such $A$ will be called a contractible subvariety of codimension 1 for $f_{m}$. This causes some problems. For this we have the following result.

THEOREM 2. Let $c_{3}$ be the constant, which can be determined by $P(x)$ alone, such that $c_{3} \geqq c_{2}$ and that for $m \geqq c_{3}$,

$$
P^{\prime}\left(m\left(m_{0}-c_{0}\right)+c_{0}\right)-2 \sum_{1}^{n-1} d m^{i}\left(m_{0}+\xi / d\right)^{i}-n>0 .
$$

Let $c_{4}=\sum_{1}^{n-1} d c_{s}^{i}\left(m_{0}+\xi / d\right)^{i}+1$. Then there is a submodule $M$ of $H^{0}\left(V, \mathscr{L}\left(c_{4} c_{3} Y\right)\right), Y=m_{0} X+K(V)$, such that the rational map defined by the module $M$ is a birational transformation of $V$ and that it has no contractible subvariety of codimension 1 on $V$.

Let us denote by $\mathscr{E}_{V}$ the set of birational transformations of $V$ into a projective space which is defined by a submodule of $H^{0}\left(V, \mathscr{L}\left(c_{4} c_{3} Y\right)\right)$, 
without contractible subvariety of codimension 1 on $V$. Whenever $g \in \mathscr{E}_{V}$, we have the following result.

Proposition. $\operatorname{deg}(g(V)) \leqq c_{5}-(n-1)$ and $g(V)$ is a subvariety of the projective space of dimension $c_{5}$, where $c_{5}$ is given by

$$
c_{5}=d c_{4}^{n} c_{3}^{n}\left(m_{0}+\xi / d\right)^{n}+n-1 .
$$

Let us denote by $\Sigma_{P}$ the set of pairs $(V, X)$ of a complete nonsingular variety $V$ and a nondegenerate $V$-divisor $X$ such that

$$
\chi(V, \mathscr{L}(m X))=P(m) .
$$

The above results in the Proposition show that the set of $g(V), g \in \mathscr{E}_{V}$, as $(V, X)$ ranges in $\Sigma_{P}$, is covered by a finite union of irreducible algebraic families of nonsingular subvarieties in the projective space of dimension $c_{5}$. Cutting down this finite union of irreducible algebraic families as much as possible by imposing algebro-geometric conditions satisfied by $g(V)$, $g \in \mathscr{E}_{V}$ and $(V, X) \in \Sigma_{P}$, we show with a slight technical modification that the set of $g(V)$ forms a finite union of irreducible algebraic families of nonsingular subvarieties of the projective space. From these last families we construct a finite union $F$ of irreducible algebraic families which satisfies the following properties:

(i) A member $U$ of $F$ is a nonsingular subvariety of a projective space.

(ii) When we polarize $U$ so that a hyperplane section is a polar divisor, the Hilbert characteristic polynomial of this polarized variety is $P(x)$.

(iii) When $(V, X) \in \Sigma_{P}$, there is a member $U$ of $F$ and an isomorphism $h$ of $V$ on $U$ such that $h(r X)$ is a hyperplane section of $U$ for some positive integer $r$.

From this we get the following main theorem at once.

THEOREM 3. Let $(V, X)$ be a pair of a complete nonsingular variety and a nondegenerate $V$-divisor $X$. Let $P(m)=\chi(V, \mathscr{L}(m X))$. Then there is a constant $c$, which depends only on $P(x)$, such that for $m \geqq c$, the module $H^{0}(V, \mathscr{L}(m X))$ defines a projective embedding of $V$.

\section{REFERENCES}

1. M. Artin, The implicit function theorem in algebraic geometry, Algebraic Geometry, Papers presented at the Bombay Colloquium, Oxford, Bombay, 1969, pp. 13-34.

2. W. L. Baily, On the moduli of Jacobian varieties, Ann. of Math. (2) 71 (1960), 303-314. MR 22 \#1583.

3. - On the theory of $\theta$-functions, the moduli of Abelian varieties and the moduli of curves, Ann. of Math. (2) 75 (1962), 342-381. MR 29 \#103.

4. W. L. Chow-v.d. Waerden, Zur Algebraischen Geometrie. IX, Math. Ann. 113 (1937), 692-704. 
5. A. Grothendieck and J. Dieudonné, Éléments de géometrie algébriques, Inst. Hautes Études Sci. Publ. Math. (1960 ff)

6. K. Kodaira, On a differential-geometric method in the theory of analytic stacks, Proc. Nat. Acad. Sci. U.S.A. 39 (1953), 1268-1273. MR 16, 618.

7. K. Kodaira and D. C. Spencer, On deformations of complex analytic structures. I, II, Ann. of Math. (2) 67 (1958), 328-466. MR 22 \#3009.

8. S. Lefschetz, On certain numerical invariants of algebraic varieties, with applications to Abelian varieties, Trans. Amer. Math. Soc. 22 (1921), 327-482.

9. T. Matsusaka, Theory of Q-varieties, The Mathematical Society of Japan, Tokyo, 1964. MR 32 \#4131.

10. - Polarized varieties, fields of moduli and generalized Kummer varieties of polarized abelian varieties, Amer. J. Math. 80 (1958), 45-82. MR 20 \#878.

11. — Algebraic deformations of polarized varieties, Nagoya Math. J. 31 (1968), 185-245. MR 41 \#3473.

12. - On canonically polarized varieties. II, Amer. J. Math. 92 (1970), 283-292. MR 41 \#8415b.

13. _ Polarized varieties with a given Hilbert characteristic polynomial, Amer. J. Math. 94 (1972), 1027-1077.

14. T. Matsusaka and D. Mumford, Two fundamental theorems on deformations of polarized varieties, Amer. J. Math. 86 (1964), 668-684. MR 30 \#2005.

15. D. Mumford, Pathologies. III, Amer. J. Math. 89 (1967), 94-104. MR 36 \#182.

16. I. Satake, On the compactification of the Siegel space, J. Indian Math. Soc. 20 (1956), 259-281. MR 18, 934.

17. J.-P. Serre, Un théorème de dualité, Comment. Math. Helv. 29 (1955), 9-26. MR 16, 736.

18. C. L. Siegel, Gesammelte Abhandlungen, Bände II, Springer-Verlag, Berlin and New York, 1966. MR 33 \#5441.

19. A. Weil, Foundations of algebraic geometry, rev. ed., Amer. Math. Soc. Colloq. Publ. vol. 29, Amer. Math. Soc., Providence, R.I., 1962. MR 26 \#2439.

Department of Mathematics, Brandeis University, Waltham, Massachusetts 02154 